\title{
Effect of filters on the time-delay interferometry residual laser noise for LISA
}

\author{
Jean-Baptiste Bayle, Marc Lilley, Antoine Petiteau, and Hubert Halloin \\ APC, Université Paris Diderot, CNRS/IN2P3, CEA/Irfu, Observatoire de Paris, \\ Sorbonne Paris Cité, 10 rue Alice Domont et Léonie Duquet, 75013 Paris, France
}

(Dated: May 22, 2020)

\begin{abstract}
The Laser Interferometer Space Antenna (LISA) is a European Space Agency mission that aims to measure gravitational waves in the millihertz range. Laser frequency noise enters the interferometric measurements and dominates the expected gravitational signals by many orders of magnitude. Time-delay interferometry (TDI) is a technique that reduces this laser noise by synthesizing virtual equal-arm interferometric measurements. Laboratory experiments and numerical simulations have confirmed that this reduction is sufficient to meet the scientific goals of the mission in proof-ofconcept setups. In this paper, we show that the on-board antialiasing filters play an important role in TDI's performance when the flexing of the constellation is accounted for. This coupling was neglected in previous studies. To reach an optimal reduction level, filters with vanishing group delays must be used on board or synthesized off-line. We propose a theoretical model of the residual laser noise including this flexing-filtering coupling. We also use two independent simulators to produce realistic measurement signals and compute the corresponding TDI Michelson variables. We show that our theoretical model agrees with the simulated data with exquisite precision. Using these two complementary approaches, we confirm TDI's ability to reduce laser frequency noise in a more realistic mission setup. The theoretical model provides insight on filter design and implementation.
\end{abstract}

\section{INTRODUCTION}

The Laser Interferometer Space Antenna (LISA) is a European Space Agency (ESA) scientific space mission which aims to measure gravitational waves (GWs) in the millihertz range [1]. Those waves are predicted by Einstein's theory of general relativity and produced by the quadrupolar moment of very dense objects, such as black hole binaries or coalescing supermassive black holes. The detection of low-frequency gravitational waves will help answer numerous astrophysical, cosmological, and theoretical questions, related, for example, to the formation of black hole binaries and extreme mass ratio inspirals, the formation of galaxies or general relativity in the strong field regime [1].

The mission is expected to be launched in the year 2034. Three spacecraft will trail the Earth around the Sun, in a nearly equilateral triangular configuration with armlengths of about 2.5 million kilometers. Each spacecraft contains two free falling test masses acting as inertial sensors [1] and two optical benches. Six laser links connect the six optical benches performing interferometric measurements between the local and distant laser beams. These optical setups are capable of measuring the differential displacement between the local and remote test masses with subpicometer precision [2, 3]. In the latest design, each spacecraft performs six interferometric measurements (see section II), which are then telemetered to Earth.

Among the multiple sources of noise which enter the measurements made by LISA, laser frequency noise is dominant. Its amplitude is greater than that of other (secondary) noises and that of GWs by several orders of magnitude. The armlengths of the LISA constellation are indeed not equal, preventing laser noise to be canceled when the beams are recombined. Time-delay in- terferometry (TDI) is an algorithm first proposed by [4] that aims to reduce the laser frequency noise by 8 orders of magnitude, bringing it below secondary noises and $\mathrm{GW}$ signals [5]. TDI synthesizes virtual equal-arm interferometric measurements by combining time-shifted measurements from LISA.

Laboratory experiments have been performed to study whether TDI can be applied correctly and whether its performance meets mission requirements, in various setups. A first demonstrator was designed at the Jet Propulsion Lab [6] to reproduce noise couplings and measure TDI noise reduction in a fixed two-arm configuration. It was shown that the laser frequency noise could be reduced to the desired level. The Hexagon interferometer [7] is a metrology test bed developed at the Albert Einstein Institute, and consists of three locked lasers 8. It was used both to test the performance and feasibility of TDI for heterodyne interferometry and to test phasemeter prototypes. LISA On Table (LOT) is an electro-optical simulator developed at the laboratoire Astroparticules et Cosmologie (APC) [9, 10. The results show that in the case of two static unequal arms, first-generation TDI cancels the laser frequency noise as expected. The University of Florida LISA simulator (UFLIS) uses electronic phase delay units to simulate the time variation of the armlengths. First-generation TDI was successfully tested, reaching the required performance, while TDI 2.0 results were limited by the noise of the electronic phase delay units [11. To date, however, no realistic demonstrator using time-varying armlengths was successful in measuring the performance of TDI 2.0.

Computer simulations have also been used to check the performance of the laser frequency noise removal performed by TDI Synthetic LISA was developed by M. Vallisneri [12 to study TDI laser noise reduction for a flexing constellation, in an idealized configuration. Us- 
ing this simulator, it was shown that one must use the second-generation TDI algorithms to meet noise reduction requirements. LISACode [13, 14] is the tool currently used by the LISA Simulation Group and the LISA Data Challenge (LDC) to produce realistic data. It uses a high-level model of the instrument to reproduce the instrumental response to incoming gravitational waves. LISACode also includes models for several sources of noise, including the aforementioned laser frequency noise. LISANode [15] is a new prototype end-to-end mission simulator. It is a very flexible framework that enables the study of various instrumental configurations. LISANode includes an up-to-date model for the instrument, various sources of noise and the TDI algorithms.

In this paper, we have developed an analytic model that describes both LISA and TDI for a realistic setup in order to determine which instrumental factors play an important role in the laser frequency noise reduction performance. This model reproduces the results of both LISANode and LISACode with great precision. We include the effect of the so-called flexing, i.e. time-varying armlengths, and that of the antialiasing filtering applied before the high-frequency measurements are downsampled and telemetered to Earth. The agreement between our theoretical model and our simulations demonstrates that LISACode and LISANode are implemented correctly. Our work also shows that a coupling between the flexing of the constellation and the antialiasing filters can degrade significantly TDI]s performance. We show that the effect of this coupling is mitigated with well-designed filters and an specific off-line treatment. The remaining laser frequency noise can then be maintained below mission requirements in the frequency band between $10^{-4}$ and $10^{-1} \mathrm{~Hz}$, for second-generation TDI

We first present the LISA mission setup that was modeled analytically and simulated numerically in section II. and the TDI algorithm that was used in section III In section IV], we derive the corresponding analytic model for TDI 1.5 and 2.0. In section $\mathrm{V}$ we describe LISACode and LISANode, and give details about the configuration used to generate the data. Finally, in section VI, we compare and discuss the results of the simulators and of the analytic approach. In particular, we study the effect of different types of filters and discuss their potential implementations.

\section{INSTRUMENTAL SETUP}

LISA is a constellation of three spacecraft forming a nearly equilateral triangle. The constellation's center of mass trails the Earth in its orbit around the Sun by around 20 deg. [1. Each spacecraft emits and receives a laser beam along each of the two arms connecting it to its companion spacecraft. All spacecraft host two movable optical system assemblies (MOSAs) an on-board computer, a phasemeter, and two laser sources. A MOSA is composed of a telescope and an optical bench. The

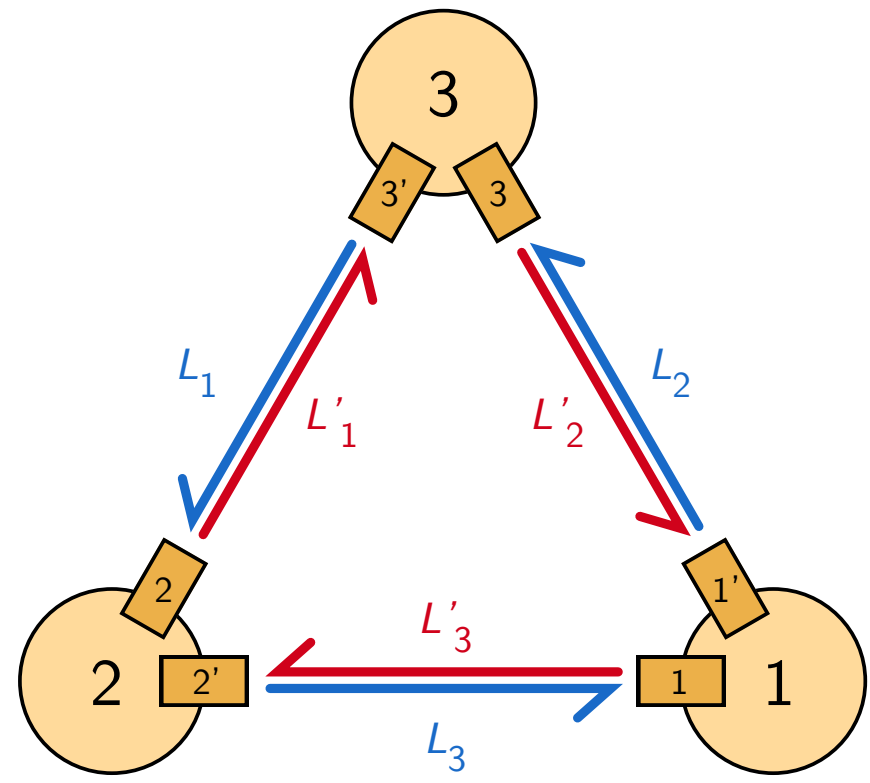

Figure 1. Conventions for labeling spacecraft, MOSAs, lasers, optical benches and arms. Primed indices are used for arms pointing clockwise, and for MOSAs and optical benches receiving light clockwise.

telescope sends an outgoing laser beam to its companion spacecraft and collects incoming light. Various conventions are used in the literature to denote the spacecraft, optical benches and arms. In this paper, we number these components according to fig. 1 .

In this paper, we consider the latest optical design, often called split interferometry. It is extensively described in [16]. Three interferometric measurements are performed on each optical bench $i$ : the science $s_{i}$ (respectively, reference $\tau_{i}$ ) signal is the beat note between the distant (respectively, adjacent) and local beams without any reflection on the test masses. The test mass signal $\epsilon_{i}$ corresponds to the beat note formed by the local and adjacent beams after reflection onto the local test mass.

For simplicity, we neglect all secondary noise sources, such as the read-out noise, the optical path noise, and the test mass acceleration noise. The clocks on board each spacecraft are assumed to be perfect and we neglect both the clock noise and the sideband measurements that are used to remove parts of this noise [16, 17]. We therefore only study laser frequency noise, denoted $p_{i}(t)$ and $p_{i}^{\prime}(t)$, where $i$ is the spacecraft index. We model it here as a white noise with a power spectral density (PSD) of $10^{-26} \mathrm{~Hz}^{-1}$. Under those assumptions, split interferometry reduces to the legacy design described in [14.

The interferometric measurements are delivered by the phasemeter to the on-board computer at $20 \mathrm{~Hz}$. Due to limitations in the telemetry passband, they must be downsampled to $2 \mathrm{~Hz}$ before they are transmitted to Earth [1]. Antialiasing filters are used to prevent power folding in the band of interest during decimation. These filters are assumed to be identical on board all spacecraft, 


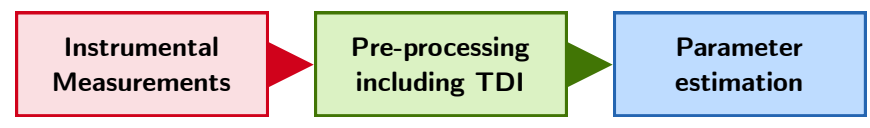

Figure 2. Main steps of the analysis chain.

and consist of a convolution with a filter kernel $f(t)$. We define the filter operator $\mathcal{F}$, such that $\mathcal{F} x(t)=(f * x)(t)$ for any signal $x(t)$.

We model all signals as Doppler observables, i.e. as the ratio of the instantaneous frequency deviation from the nominal carrier frequency $\left[\nu(t)-\nu_{0}\right] / \nu_{0}[12]$ over that nominal carrier frequency. We neglect frequency planning [18] and Doppler shifts due to the relative motion of the spacecraft. Therefore, in this paper, the carrier nominal frequency remains constant and equal for all beams, and interferometric signals are obtained by forming the difference of two incoming Doppler observables.

The propagation of laser beams between two spacecraft is modeled by applying time-varying delays. These delays correspond to the sum of all delays in the optical, analog and digital signal chains, though the main contribution remains the light travel times between the spacecraft. Therefore we suppose here that they are given by the armlengths and the speed of light in vacuum. We denote $\mathcal{D}_{i}$ the operator associated with traveling along arm $i$, of length $c \times L_{i}(t)$. For example, the laser frequency noise received by optical bench $2^{\prime}$ from laser 1 , after it has traveled along arm 3 , is given by

$$
\mathcal{D}_{3} p_{1}(t)=p_{1}\left(t-L_{3}(t)\right)
$$

We give the expressions of the measurement signals for the spacecraft 1; others are obtained by circular permutation of the indices. The science signals read

$$
\begin{aligned}
s_{1} & =\mathcal{F} \mathcal{D}_{3} p_{2}^{\prime}-\mathcal{F} p_{1}, \\
s_{1}^{\prime} & =\mathcal{F} \mathcal{D}_{2^{\prime}} p_{3}-\mathcal{F} p_{1}^{\prime} .
\end{aligned}
$$

In the absence of secondary noise sources, the expressions for the test mass and reference signals are equal and given by

$$
\begin{aligned}
\epsilon_{1} & =\tau_{1}=\mathcal{F} p_{1}^{\prime}-\mathcal{F} p_{1}, \\
\epsilon_{1}^{\prime} & =\tau_{1}^{\prime}=\mathcal{F} p_{1}-\mathcal{F} p_{1}^{\prime} .
\end{aligned}
$$

\section{TIME-DELAY INTERFEROMETRY}

TDI is a multistaged algorithm [8] which is performed off-line, before astrophysical and cosmological source parameters are extracted (cf. fig. 22). It is mainly conceived to reduce reduce laser frequency noise, but intermediary steps also cancel other instrumental sources of noise. We shall only consider laser frequency noise, but will nevertheless use the full TDI expressions in this section, for consistency with the literature.

One first uses the measurement signals to form the intermediary variables $\xi_{i}$, then $Q_{i}$ and then finally $\eta_{i}$. These combinations, respectively, cancel out the optical bench displacement noise (here set to zero), reduce the signals to one free-running laser per spacecraft and suppress clock noise (here set to zero). Note that the test mass acceleration and optical measurement system noises, also set to zero in this study, are not suppressed by these combinations. $\xi, Q$, and $\eta$ are defined in [8] and can be written under our assumptions as

$$
\begin{gathered}
\xi_{i}=s_{i}-\frac{\mathcal{D}_{k} \epsilon_{j}^{\prime}-\mathcal{D}_{k} \tau_{j}^{\prime}}{2}, \\
\xi_{i}^{\prime}=s_{i}^{\prime}-\frac{\mathcal{D}_{j^{\prime}} \epsilon_{k}-\mathcal{D}_{j^{\prime}} \tau_{k}}{2}, \\
Q_{i}=\xi_{i}+\frac{\mathcal{D}_{k} \tau_{j}^{\prime}-\mathcal{D}_{k} \tau_{j}}{2}, \\
Q_{i}^{\prime}=\xi_{i}^{\prime}+\frac{\tau_{i}^{\prime}-\tau_{i}}{2}, \\
\eta_{i}=Q_{i}, \\
\eta_{i}^{\prime}=Q_{i}^{\prime} .
\end{gathered}
$$

Next, TDI synthesizes virtual equal-arm interferometric measurements in order to reduce laser frequency noise. This is done by applying one of the appropriate sets of nested delays to the $\eta_{i}$ variables, and by combining the resulting terms. In this paper, we focus on the Michelson variables $X, Y$ and $Z$, which synthesize pairwise independent Michelson-like interferometers. There exist several generations of Michelson variables, which depend on the complexity of the spacecraft motion. TDI] version 1.0 applies to a static configuration. Version 1.5 applies to a rigid but rotating configuration. Finally, TDI version 2.0 applies to a rotating configuration with armlengths varying linearly in time. In this paper we focus on versions 1.5 and 2.0 of TDI.

The expressions for the $X$ variable for generations 1.5 and 2.0 [19] are given by

$$
\begin{aligned}
X_{1}= & \eta_{1^{\prime}}+\mathcal{D}_{2^{\prime}} \eta_{3}+\mathcal{D}_{2^{\prime} 2} \eta_{1}+\mathcal{D}_{2^{\prime} 23} \eta_{2^{\prime}}-\left(\eta_{1}+\mathcal{D}_{3} \eta_{2^{\prime}}+\mathcal{D}_{33^{\prime}} \eta_{1^{\prime}}+\mathcal{D}_{33^{\prime} 2^{\prime}} \eta_{3}\right) \\
X_{2}= & X_{1}+\mathcal{D}_{2^{\prime} 233^{\prime}} \eta_{1}+\mathcal{D}_{2^{\prime} 233^{\prime} 3} \eta_{2^{\prime}}+\mathcal{D}_{2^{\prime} 233^{\prime} 33^{\prime}} \eta_{1^{\prime}}+\mathcal{D}_{2^{\prime} 233^{\prime} 33^{\prime} 2^{\prime}} \eta_{3} \\
& -\left(\mathcal{D}_{33^{\prime} 2^{\prime} 2} \eta_{1^{\prime}}+\mathcal{D}_{33^{\prime} 2^{\prime} 22^{\prime}} \eta_{3}+\mathcal{D}_{33^{\prime} 2^{\prime} 22^{\prime} 2} \eta_{1}+\mathcal{D}_{33^{\prime} 2^{\prime} 22^{\prime} 23} \eta_{2^{\prime}}\right)
\end{aligned}
$$

where we have used the nested delay notation $\mathcal{D}_{i_{1} i_{2} \ldots i_{n}} \equiv \mathcal{D}_{i_{1}} \mathcal{D}_{i_{2}} \ldots \mathcal{D}_{i_{n}}$. The remaining Michelson variables $Y$ and $Z$ can be obtained by circular permutation of the indices.

Substituting in eqs. (5) and (6) the values for $\eta$ and $\eta^{\prime}$, given by eq. (4), yields expressions which depend on laser frequency noises only. Let us first neglect the filters, i.e. we set $\mathcal{F}=1$. Because we are dealing with time-varying 
armlengths, the Michelson variables can be expressed with nonvanishing delay commutators $[A, B]=A B-B A$,

$$
\begin{aligned}
& X_{1}=\left(\left[\mathcal{D}_{2^{\prime} 2}, \mathcal{D}_{3}\right] \mathcal{D}_{3^{\prime}}+\mathcal{D}_{3}\left[\mathcal{D}_{2^{\prime} 2}, \mathcal{D}_{3^{\prime}}\right]\right) p_{1} \\
& X_{2}=\left(\left[\mathcal{D}_{2^{\prime} 2}, \mathcal{D}_{33^{\prime}}\right] \mathcal{D}_{33^{\prime 2} 2^{\prime 2}}-\mathcal{D}_{33^{\prime} 2^{\prime 2} 2}\left[\mathcal{D}_{2^{\prime} 2}, \mathcal{D}_{33^{\prime}}\right]\right) p_{1}
\end{aligned}
$$

If we now include the effect of the filter, delay-filter commutators appear and the residual laser frequency noise now reads

$$
\begin{aligned}
X_{1}= & \left(\left[\mathcal{D}_{2^{\prime} 2}, \mathcal{D}_{3}\right] \mathcal{F} \mathcal{D}_{3^{\prime}}+\mathcal{D}_{3}\left[\mathcal{D}_{2^{\prime} 2}, \mathcal{D}_{3^{\prime}}\right] \mathcal{F}\right) p_{1} \\
& +\mathcal{D}_{3}\left(1-\mathcal{D}_{2^{\prime} 2}\right)\left[\mathcal{D}_{3^{\prime}}, \mathcal{F}\right] p_{1}+\left(1-\mathcal{D}_{33^{\prime}}\right) \mathcal{D}_{2^{\prime}}\left[\mathcal{F}, \mathcal{D}_{2}\right] p_{1^{\prime}} \\
& +\left(1-\mathcal{D}_{2^{\prime} 2}\right)\left[\mathcal{D}_{3}, \mathcal{F}\right] p_{2^{\prime}}+\left(1-\mathcal{D}_{33^{\prime}}\right)\left[\mathcal{D}_{2^{\prime}}, \mathcal{F}\right] p_{3}, \\
X_{2}= & \left(\left[\mathcal{D}_{2^{\prime} 2}, \mathcal{D}_{33^{\prime}}\right] \mathcal{D}_{33^{\prime} 2^{\prime} 2} \mathcal{F}+\mathcal{D}_{33^{\prime} 2^{\prime} 2}\left[\mathcal{D}_{33^{\prime}}, \mathcal{D}_{2^{\prime} 2}\right] \mathcal{F}\right) p_{1} \\
& +\left(1-\mathcal{D}_{2^{\prime} 2}-\mathcal{D}_{2^{\prime} 233^{\prime}}+\mathcal{D}_{33^{\prime} 2^{\prime} 22^{\prime} 2}\right) \mathcal{D}_{3}\left[\mathcal{D}_{3^{\prime}}, \mathcal{F}\right] p_{1} \\
& +\left(1-\mathcal{D}_{33^{\prime}}-\mathcal{D}_{33^{\prime} 2^{\prime} 2}+\mathcal{D}_{2^{\prime} 233^{\prime} 33^{\prime}}\right) \mathcal{D}_{2^{\prime}}\left[\mathcal{F}, \mathcal{D}_{2}\right] p_{1^{\prime}} \\
& +\left(1-\mathcal{D}_{2^{\prime} 2}-\mathcal{D}_{2^{\prime} 233^{\prime}}+\mathcal{D}_{33^{\prime} 2^{\prime} 22^{\prime} 2}\right)\left[\mathcal{D}_{3}, \mathcal{F}\right] p_{2^{\prime}} \\
& +\left(1-\mathcal{D}_{33^{\prime}}-\mathcal{D}_{33^{\prime} 2^{\prime} 2}+\mathcal{D}_{2^{\prime} 233^{\prime} 33^{\prime}}\right)\left[\mathcal{F}, \mathcal{D}_{2^{\prime}}\right] p_{3} .
\end{aligned}
$$

In the next section, we use these expressions to derive an analytic model for the residual laser frequency noise after application of TDI. In section V, we numerically simulate the measurement signals, generate the $X, Y$, and $Z$ variables, and estimate their PSDs.

\section{ANALYTIC MODELING FOR LINEAR ARMLENGTHS}

For realistic spacecraft orbits computed using Kepler's laws [20, 21], LISA armlengths are not constant, but modulated with a characteristic time scale of a year. In this section, we expand these armlengths to first order in time. This is a good approximation of the true orbits on a scale of days, i.e. to a frequency of the order of $10^{-5} \mathrm{~Hz}$, while the lowest frequency in LISA s band of interest is $2 \times 10^{-5} \mathrm{~Hz}[22$. Therefore, deviations are expected to appear only at low frequencies.

We define the armlengths as $L_{i}(t)=L_{i}+\dot{L}_{i} t$, where $L_{i}$ and $\dot{L}_{i}$ are constant. The delay operators $\mathcal{D}_{i}$ applied to the laser frequency noise $p(t)$ is now a pure delay and a time rescaling. It reads

$$
\left.\mathcal{D}_{i} p(t)=p\left[\left(1-\dot{L}_{i}\right) t-L_{i}\right)\right]
$$

Its Fourier transform is given by

$$
\frac{1}{1-\dot{L}_{i}} \exp \left(-j \omega \frac{L_{i}}{1-\dot{L}_{i}}\right) \widetilde{x}\left(\frac{\omega}{1-\dot{L}_{i}}\right)
$$

where $j^{2}=-1$ is the unit imaginary number.

\section{A. Delay commutators}

Equations (7) and (10) are written as sums of delay and delay-filter commutators. In order to compute the
PSD of these expressions, let us first derive their Fourier transforms.

The expression of nested delay operators in the time domain is deduced by repeated use of eq. (1). One finds

$$
\mathcal{D}_{i_{1} \ldots i_{n}} x(t)=x\left(S_{n} t-\sum_{k=1}^{n} S_{k-1} L_{i_{k}}\right)
$$

where we have defined the product $S_{k}=\prod_{p=1}^{k}\left(1-\dot{L}_{i_{p}}\right)$ for $k>0$, and $S_{0}=1$. The Fourier transform of eq. (13) is given by

$$
\frac{1}{S_{n}} \exp \left(-j \omega \sum_{k=1}^{n} \frac{L_{i_{k}}}{S_{k}}\right) \widetilde{x}\left(\frac{\omega}{S_{n}}\right)
$$

Let us now consider the commutator of $n$ delay operators applied to a signal $x(t)$, which we denote $y(t)=\left[\mathcal{D}_{i_{1} \ldots i_{m}}, \mathcal{D}_{i_{m+1} \ldots i_{n}}\right] x(t)=\mathcal{D}_{i_{1}} \ldots \mathcal{D}_{i_{n}} x(t)-$ $\mathcal{D}_{i_{m+1}} \ldots \mathcal{D}_{i_{n}} \mathcal{D}_{i_{1}} \ldots \mathcal{D}_{i_{m}} x(t)$ in the following. Using eq. (13) and after some work on the indices, it is possible to express it as

$$
\begin{aligned}
y(t) & =x\left(S_{n} t-\sum_{k=1}^{n} S_{k-1}^{i} L_{i_{k}}\right) \\
& -x\left(S_{n} t-\frac{S_{n}^{i}}{S_{m}^{i}} \sum_{k=1}^{m} S_{k-1}^{i} L_{i_{k}}-\frac{1}{S_{m}^{i}} \sum_{k=m+1}^{n} S_{k-1}^{i} L_{i_{k}}\right) .
\end{aligned}
$$

We can expand this expression to first order in powers of the armlength derivatives $\dot{L}_{i}$. If we moreover assume that all armlengths at $t=0$ are almost equal, i.e. $L_{i} \approx L$ for all $i$, it reads

$$
y(t) \approx L\left[(n-m)\left(\sum_{k=1}^{m} \dot{L}_{i_{k}}\right)-m\left(\sum_{k=m+1}^{n} \dot{L}_{i_{k}}\right)\right] \frac{\mathrm{d} x}{\mathrm{~d} t}(t-n L) .
$$


The corresponding Fourier transform $\widetilde{y}(\omega)$ is

$$
\begin{aligned}
\widetilde{y}(\omega) & \approx-j \omega L e^{-j \omega n L} \\
& \times\left[(n-m)\left(\sum_{k=1}^{m} \dot{L}_{i_{k}}\right)-m\left(\sum_{k=m+1}^{n} \dot{L}_{i_{k}}\right)\right] \widetilde{x}(\omega) .
\end{aligned}
$$

\section{B. Delay-filter commutators}

In the time domain, the application of the filter $\mathcal{F}$ on a signal $x(t)$ is written as the convolution of the latter and the filter's kernel $f$. In frequency domain, this translates into the product $\widetilde{x}(\omega) \widetilde{f}(\omega)$.

Therefore, if we apply the filter after a series of delays $\mathcal{F D}_{i_{1}} \ldots \mathcal{D}_{i_{n}} x(t)$, we have, in Fourier domain,

$$
\frac{1}{S_{n}} \exp \left(-j \omega \sum_{k=1}^{n} \frac{L_{i_{k}}}{S_{k}}\right) \widetilde{x}\left(\frac{\omega}{S_{n}}\right) \tilde{f}(\omega)
$$

If the filter is applied before the delays $\mathcal{D}_{i_{1}} \ldots \mathcal{D}_{i_{n}} \mathcal{F} x(t)$, we now have

$$
\frac{1}{S_{n}} \exp \left(-j \omega \sum_{k=1}^{n} \frac{L_{i_{k}}}{S_{k}}\right) \widetilde{x}\left(\frac{\omega}{S_{n}}\right) \tilde{f}\left(\frac{\omega}{S_{n}}\right)
$$

Let us define the signal $y(t)$ as the commutator of nested delays $\mathcal{D}_{i_{1}} \ldots \mathcal{D}_{i_{n}}$ and a filter $\mathcal{F}$. In general,

$$
\begin{aligned}
y(t) & =\left[\mathcal{D}_{i_{1}} \ldots \mathcal{D}_{i_{n}}, \mathcal{F}\right] x(t) \\
& =\mathcal{D}_{i_{1}} \ldots \mathcal{D}_{i_{n}} \mathcal{F} x(t)-\mathcal{F} \mathcal{D}_{i_{1}} \ldots \mathcal{D}_{i_{n}} x(t)
\end{aligned}
$$

Using the previous equations, the exact expression in Fourier space writes

$$
\begin{aligned}
& \widetilde{y}(\omega)=\frac{1}{S_{n}} \exp \left(-j \omega \sum_{k=1}^{n} \frac{L_{i_{k}}}{S_{k}}\right) \\
& \times \widetilde{x}\left(\frac{\omega}{S_{n}}\right)\left[\tilde{f}\left(\frac{\omega}{S_{n}}\right)-\tilde{f}(\omega)\right] .
\end{aligned}
$$$$
S_{X_{1}} \approx 8 S_{p} \omega^{2}\left[2 L^{2}\left(\dot{L}_{2}-\dot{L}_{3}\right)^{2} S_{f}(\omega)-L \dot{L}_{3}\left(\dot{L}_{2}-\dot{L}_{3}\right) D_{\mathcal{F}}(\omega)+\sin ^{2}(\omega L)\left(\dot{L}_{2}^{2}+\dot{L}_{3}^{2}\right) K_{\mathcal{F}}(\omega)\right] \text {, }
$$

If we use a first-order expansion in the armlength derivatives $\dot{L}_{i}$, the previous equation reads

$$
\widetilde{y}(\omega) \approx \omega \exp \left(-j \omega \sum_{k=1}^{n} L_{i_{k}}\right)\left(\sum_{k=1}^{n} \dot{L}_{i_{k}}\right) \frac{\mathrm{d} \tilde{f}}{\mathrm{~d} \omega}(\omega) \widetilde{x}(\omega)
$$

One can note the linear dependency on the angular frequency, and the first-order factor $\sum_{k=1}^{n} \dot{L}_{i_{k}}$. The term of interest here is $\frac{\mathrm{d} \tilde{f}}{\mathrm{~d} \omega}(\omega)$, which depends on the filter characteristics.

\section{Residual laser noise}

First, let us neglect the filters; i.e., we set $\mathcal{F}=1$. We substitute in eq. (7) the first-order expression for the delay commutator given in eq. 17]. This yields the approximated Fourier transform of the residual laser noise for $X_{1}$.

As expected, first-order terms vanish for TDI 2.0. We expand eq. (8) to second order, using the exact expression of the delay commutator given in eq. (17). This yields the approximated Fourier transform of the residual laser noise for $X_{2}$.

The corresponding $\mathrm{PSD}$ are obtained by taking the squared modulus and the ensemble average of the Fourier transforms. We use the fact that the laser noises have zero mean, i.e. $\left\langle\widetilde{p}_{i}(\omega)\right\rangle=0$ for all $i$. In addition, different laser noises are uncorrelated, i.e. $\left\langle\widetilde{p_{i}}\left(\omega_{1}\right) \widetilde{p_{j}}\left(\omega_{2}\right)\right\rangle=0$ if $i \neq j$. They all are white noises with the same constant $\mathrm{PSD}$, denoted $S_{p}$. We have

$$
\begin{aligned}
& S_{X_{1}}(\omega) \approx 16 S_{p} \omega^{2} L^{2}\left(\dot{\dot{L}_{2}}-\dot{\dot{L}_{3}}\right)^{2} \\
& S_{X_{2}}(\omega) \approx 64 S_{p} \omega^{2} L^{2}\left({\dot{L_{2}}}^{2}-{\dot{L_{3}}}^{2}\right)^{2} .
\end{aligned}
$$

As expected, the residual laser noise scales with the laser frequency noise $S_{p}$, and vanishes if $\dot{L}_{1}=\dot{L}_{2}$, i.e. if the constellation undergoes a homothetic transformation.

We now introduce the effect of the filters. Using eqs. (9) and (22), we find that the first-order expansion of the laser noise residuals in $X_{1}$ is given by
Comparing this expression with eq. $(23)$, we see that an extra term of the same order appears. It corresponds to a coupling between the antialiasing filters and the time-varying armlengths, with a dependance on the filter characteristics expressed by the filter term $K_{\mathcal{F}}(\omega)$ and $D_{\mathcal{F}}(\omega)$, discussed below. This flexing-filtering coupling is however smaller than the previous term by a factor 
of $1 / L$, and eq. 23 still gives a good estimate for the residual laser noise in $X_{1}$.

Similarly, we use eq. 22 in eq. 10 to obtain the approximated expression for the laser noise residuals in $X_{2}$, which reads

$$
\begin{aligned}
S_{X_{2}}(\omega) \approx & 32 S_{p} \omega^{2} \sin ^{2}(\omega L) \sin ^{2}(2 \omega L) \\
& \times\left({\dot{\dot{L}_{2}}}^{2}+{\dot{L_{3}}}^{2}\right) K_{\mathcal{F}}(\omega) .
\end{aligned}
$$

We see that the flexing-filtering coupling is dominant for second-generation TDI. The level of residual laser frequency noise in $X_{2}$ is therefore strongly dependent on the filter design. We study various filters in the next paragraphs.

\section{Filter term $K_{\mathcal{F}}(\omega)$}

In the current baseline, all antialiasing filters are identical and correspond to a causal symmetrical finite impulse response (FIR) filter. Its corner frequencies are slightly below $1 \mathrm{~Hz}$, and a high attenuation must be reached for frequencies higher than $2 \mathrm{~Hz}$.

We can write the filter output $y_{n}$ as a function of the past input samples $x_{n-k}$ and $2 N+1$ coefficients $\alpha_{k}$

$$
y_{n}=\sum_{k=0}^{2 N} \alpha_{k} x_{n-k} .
$$

Its transfer function reads

$$
\widetilde{f}(\omega)=\sum_{k=0}^{2 N} \alpha_{k} e^{-j k \omega / f_{s}},
$$

$f_{s}$ is the sampling frequency. Taking its derivative with respect to the angular frequency $\omega$ immediately yields the associated filter term

$$
K_{\mathcal{F}}^{\text {causal }}(\omega)=f_{s}^{-2}\left|\sum_{k=1}^{2 N} k \alpha_{k} e^{-j k \omega / f_{s}}\right|^{2} .
$$

This causal filter has a nonvanishing group delay of $N f_{s}^{-1}$, which is responsible for the nonvanishing zerothorder term $K_{\mathcal{F}}^{\text {causal }}(\omega) \approx f_{s}^{-2}\left|\sum_{k=1}^{2 N} k \alpha_{k}\right|^{2}$, for $\omega \ll 2 \pi f_{s}$. The equivalent noncausal filter has a vanishing group delay, and the associated filter term reads

$$
K_{\mathcal{F}}^{\text {acausal }}(\omega)=4 f_{s}^{-2}\left|\sum_{k=1}^{N} k \alpha_{N+k} \sin \left(\frac{k \omega}{f_{s}}\right)\right|^{2} .
$$

We are now left with a second-order term in $\omega / 2 \pi f_{s}$, and $K_{\mathcal{F}}^{\text {acausal }}(\omega) \approx 4 \omega^{2} f_{s}^{-4}\left|\sum_{k=1}^{N} k^{2} \alpha_{N+k}\right|^{2}$.

We expect smaller laser noise residuals for noncausal filters in the LISA frequency band of interest, i.e. below $1 \mathrm{~Hz}$. This is verified in fig. 3. where causal and noncausal filter terms are plotted, for the filter used in simulations and described in section $\mathrm{V}$.

For reference, we also plotted the filter term for an infinite impulse response (IIR) (i.e. recursive) elliptic filter with the same characteristic $\$$. We see that it is larger than that of the noncausal FIR filters, which leads to larger residual noise.

\section{SIMULATIONS FOR KEPLERIAN ORBITS}

In this section, we present LISACode and LISANode, the two simulation softwares that were used to generate LISA measurement signals and process them using the TDI algorithm introduced in section III. The results are presented and discussed in section VI

LISACode is the simulator currently used by the LISA community to generate realistic data while LISANode is the baseline prototype for an end-to-end mission performance simulator. They both perform computations in time domain and produce time series for any choice of TDI variables. In the following, we only consider the Michelson variables $X, Y$, and $Z$.

Two sampling frequencies are used in our simulations. The physical sampling frequency applies to the physical subsystems in the simulators: generation of instrumental noise, beam propagation, and optical measurements. It is taken to be equal to $f_{\text {phy }}=20 \mathrm{~Hz}$ in both simulators. The interferometric signals $s_{i}, \epsilon_{i}$ and $\tau_{i}$ are downsampled to the measurement frequency $f_{\text {meas }}=2 \mathrm{~Hz}$ by means of a decimation algorithm. All preprocessing steps, including [TDI] are therefore carried out at this measurement frequency. All signals are implemented as doubles (64-bit floating-point numbers).

In both simulators, we use a symmetric FIR antialiasing filter of order 253, designed with a Kaiser window. The coefficient: $2^{2}$ are calculated such that the signal is attenuated by $240 \mathrm{~dB}$ between $0.2 \mathrm{~Hz}$ and $0.9 \mathrm{~Hz}$, and we authorize a maximum ripple of $0.1 \mathrm{~dB}$ below $0.2 \mathrm{~Hz}$. We implement filters using a direct form I and therefore, account group delays when they are not vanishing.

The propagation of the laser beams between the spacecraft is implemented using time-varying delays. Those delays are computed from the relative positions of the spacecraft, themselves deduced from their Keplerian orbits presented in [20]. These orbits include the Sagnac effect, as well as first order relativistic corrections.

All delay operators are implemented using Lagrange interpolating polynomials of order 31 . This choice is the result of a trade-off: it allows for good precision and limits execution time and numerical errors. As seen above, the TDI algorithm requires the application of multiple delay operators to the interference measurements for the

\footnotetext{
${ }^{1}$ Coefficients of the filter are given in appendix A

${ }^{2}$ Given in appendix B
} 


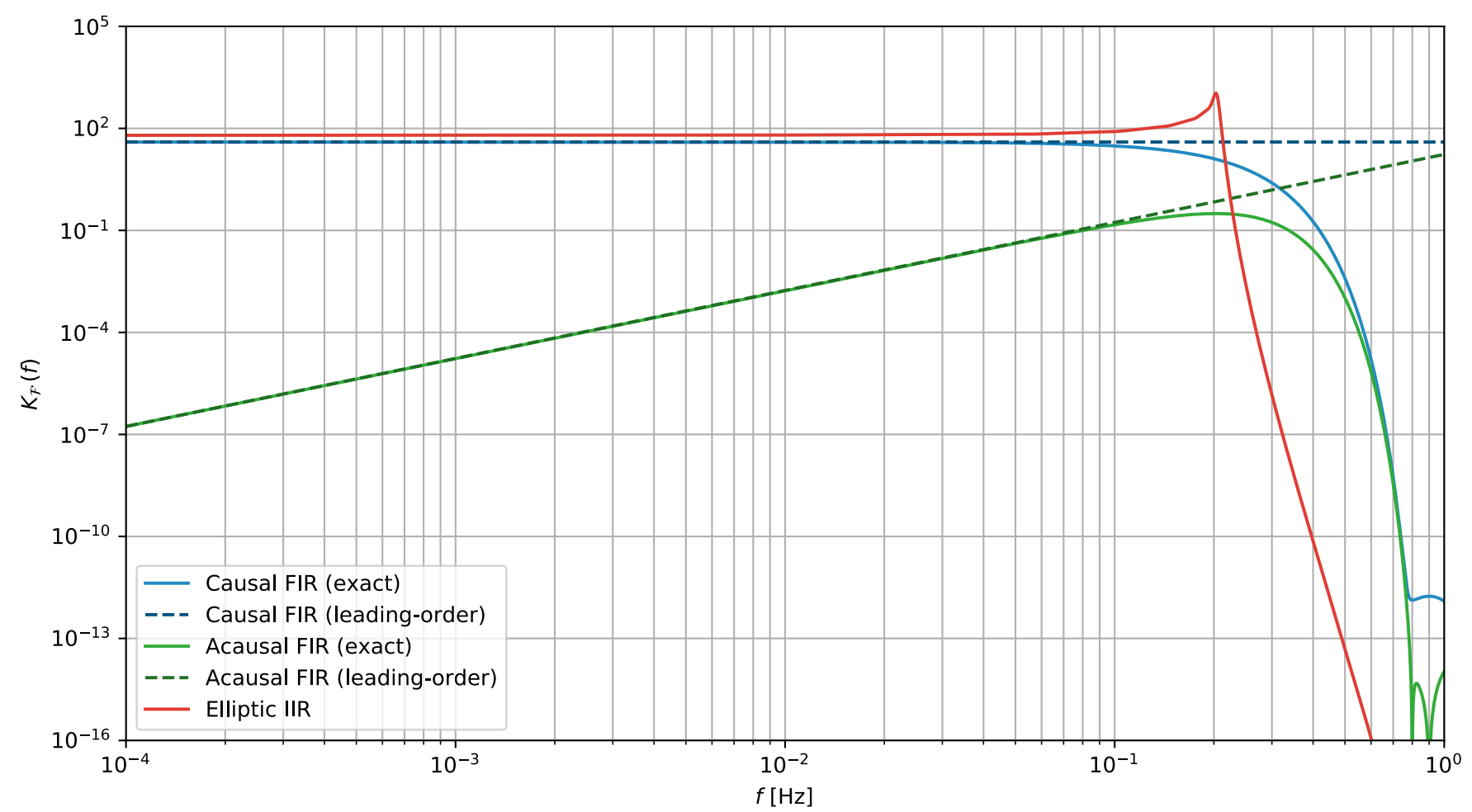

Figure 3. Levels for different filter terms $K_{\mathcal{F}}$. Dotted lines correspond to leading-order expansions.

calculation of the Michelson variables $X, Y$, and $Z$. In order to minimize the error introduced by the associated interpolations, we use a nested delay algorithm in which a single interpolation is necessary.

\section{A. LISACode}

LISACode is a high-level simulator [13, entirely written in $\mathrm{C++}$. It was used to produce noise time series for the past Mock LISA Data Challenges (MLDCs) [23] as well as for the current [LDC] It also constitutes a useful tool for the various studies of the instrument noise budget.

LISACode is based on the original optical design, equivalent, under our assumptions, to the split interferometry design described in section II] Each of the three spacecraft of the LISA constellation contains two independent lasers and two optical benches. Each optical bench holds a science and a reference interferometer; the corresponding beat notes are filtered and decimated to produce the respective measurement signals $s_{i}(t)$ and $\tau_{i}(t)$, as presented in fig. 4

The LISACode results use a $10^{7} \mathrm{~s}$ time series generated with version 2.12 .

\section{B. LISANode}

LISANode [15] is a flexible simulation tool based on the foundations of LISACode, which aims to assess [LISA]s

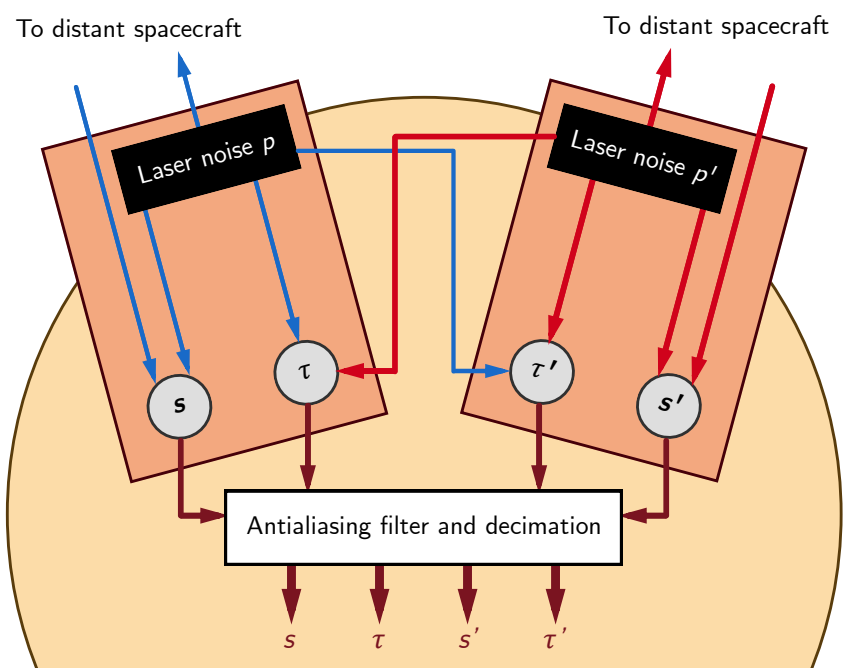

Figure 4. Original optical design used in LISACode simulations. Four interferometric measurements per spacecraft are performed: the science signals $s_{i}$ and $s_{i}^{\prime}$, along with the reference signals $\tau_{i}$ and $\tau_{i}^{\prime}$.

scientific performance. It is the current prototype for an end-to-end simulator of the mission. It was originally developed by the authors and is now part of the LISA Simulation Group activities.

Similarly to LISACode, LISANode works exclusively in the time domain so that it can handle nonlinear artifacts and produce output in the form of time series. It is based 


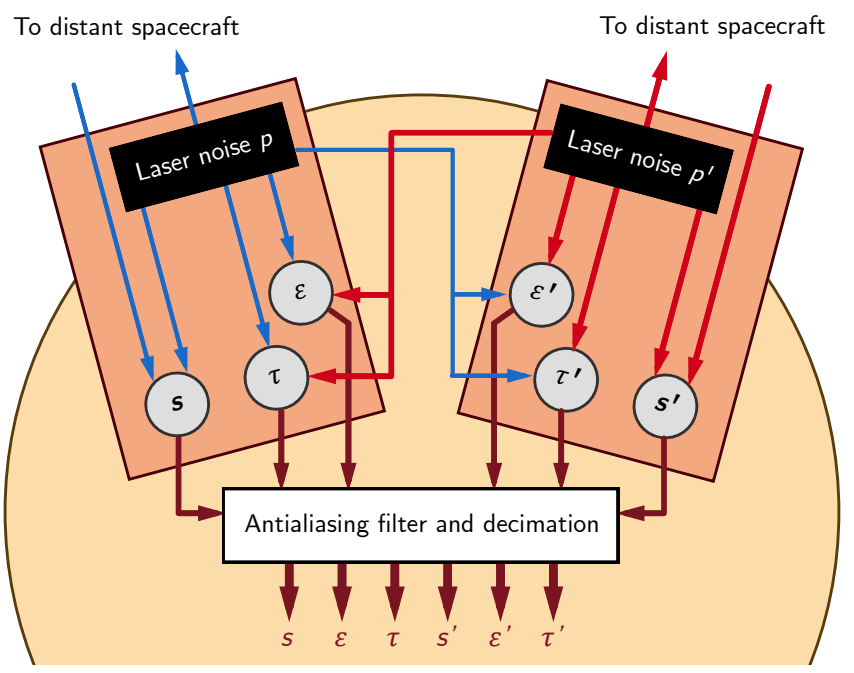

Figure 5. New split interferometry optical design used in LISANode simulations. Six interferometric measurements per spacecraft are performed: the science signals $s_{i}$ and $s_{i}^{\prime}$, the test mass signals $\epsilon_{i}$ and $\epsilon_{i}^{\prime}$, along with the reference signals $\tau_{i}$ and $\tau_{i}^{\prime}$.

on simulation graphs, written in Python, which are composed of atomic C++ computation units called nodes. A scheduler triggers node execution in a specific order and pushes data from one node to the next. In this manner, execution time is optimized and data flow is synchronized. Graphs can be nested to represent whole subsystems as one object, allowing for a high level of modularity and maintainability.

LISANode implements the newest split interferometry optical setup described in section II, and presented in fig. 5. Three interferometric measurements $s_{i}, \tau_{i}$ and $\epsilon_{i}$ (respectively, the science, test mass, and reference signals) are formed and relevant sources of noise are added to the measurements. These signals are then transmitted to the on-board computer, which contains the antialiasing filter and decimation nodes. The results of these operations are used to form the TDI Michelson variables $X$, $Y$ and $Z$.

The results use a $10^{7} \mathrm{~s}$ time series generated with LISANode version 1.1.

\section{RESULTS AND DISCUSSION}

\section{A. Results}

In figs. 6 and 7, we present the PSDs of the residual laser frequency noise for the TDI Michelson variables $X_{1}$ and $X_{2}$. We show the results of LISANode simulations for both the causal and the noncausal versions of the same filter, as described in section V) (light and dark blue curves). We plot the results of LISACode simulations for the causal filter only, in order to validate the new simulator (light orange curve). The models derived in section IV are superimposed (dashed light and dark green curves).

For reference, the red solid curves show the residual secondary noises in both $X_{1}$ and $X_{2}$ channels, simulated using LISANode and the noncausal antialiasing filter. To generate those signals, we did not change the simulation parameters. However, laser frequency noise is set to zero while the test mass acceleration (TM), optical read-out (RO) and optical path (OP) noise amplitudes were given their nominal LISA instrument noise budget values. The spectral shapes of these three secondary sources of noise are given in [22, and read

$$
\begin{aligned}
S_{\mathrm{TM}}= & \left(2.4 \times 10^{-15}\right)^{2} \\
& \times\left[1+\left(\frac{4 \times 10^{-4}}{f}\right)^{2}\right] \mathrm{m}^{2} \mathrm{~s}^{-4} \mathrm{~Hz}^{-1}, \\
S_{\mathrm{RO}}+ & S_{\mathrm{OP}}=1 \times 10^{-24} \mathrm{~m}^{2} \mathrm{~Hz}^{-1} .
\end{aligned}
$$

Because TDI does not suppress those secondary noises, but only modulates their spectra, they are used as a benchmark.

We use Welch's method to estimate the spectra, implemented with standard Python tools included in the scipy.signal module, version 1.1.0. We use segments of 40000 samples and a Nutall4 window function. The results are presented for the frequency band from $10^{-4} \mathrm{~Hz}$ to $1 \mathrm{~Hz}$.

We can see that the results of both simulators are in very good agreement. The fact that both simulators give similar results, although they use different implementations, increases our confidence in the results they produce. Note that at frequencies greater than $4 \times 10^{-1} \mathrm{~Hz}$, one observes a slight discrepancy between LISANode and LISACode. This discrepancy is due to different implementations of the Lagrange interpolating polynomials in the two simulators.

We can also observe that our analytic models match the simulated data with exquisite precision in most of the LISA band. At high frequencies, the model is no longer valid, since it does not include the errors from Lagrange interpolations. These errors, visible in the simulated data, manifest themselves by an increased level of residual laser frequency noise around $6 \times 10^{-1} \mathrm{~Hz}$. It can be shown that varying the interpolation order changes the amplitude of this effect. At lower frequencies, the simulated data deviate away from the analytic model. This is because assuming that the armlengths are varying linearly in time is only valid for frequencies higher than $1 \mathrm{mHz}$. However, we see that at these lower frequencies the residual laser noise is in any case well below mission noise level requirements.

It is also very clear that using a noncausal filter decreases significantly the residual laser noise. This effect is particularly obvious at low frequencies, as the leadingorder expansion of the filter term is constant for the causal filter, while being proportional to $\omega^{2}$ for its noncausal version; see section IV 


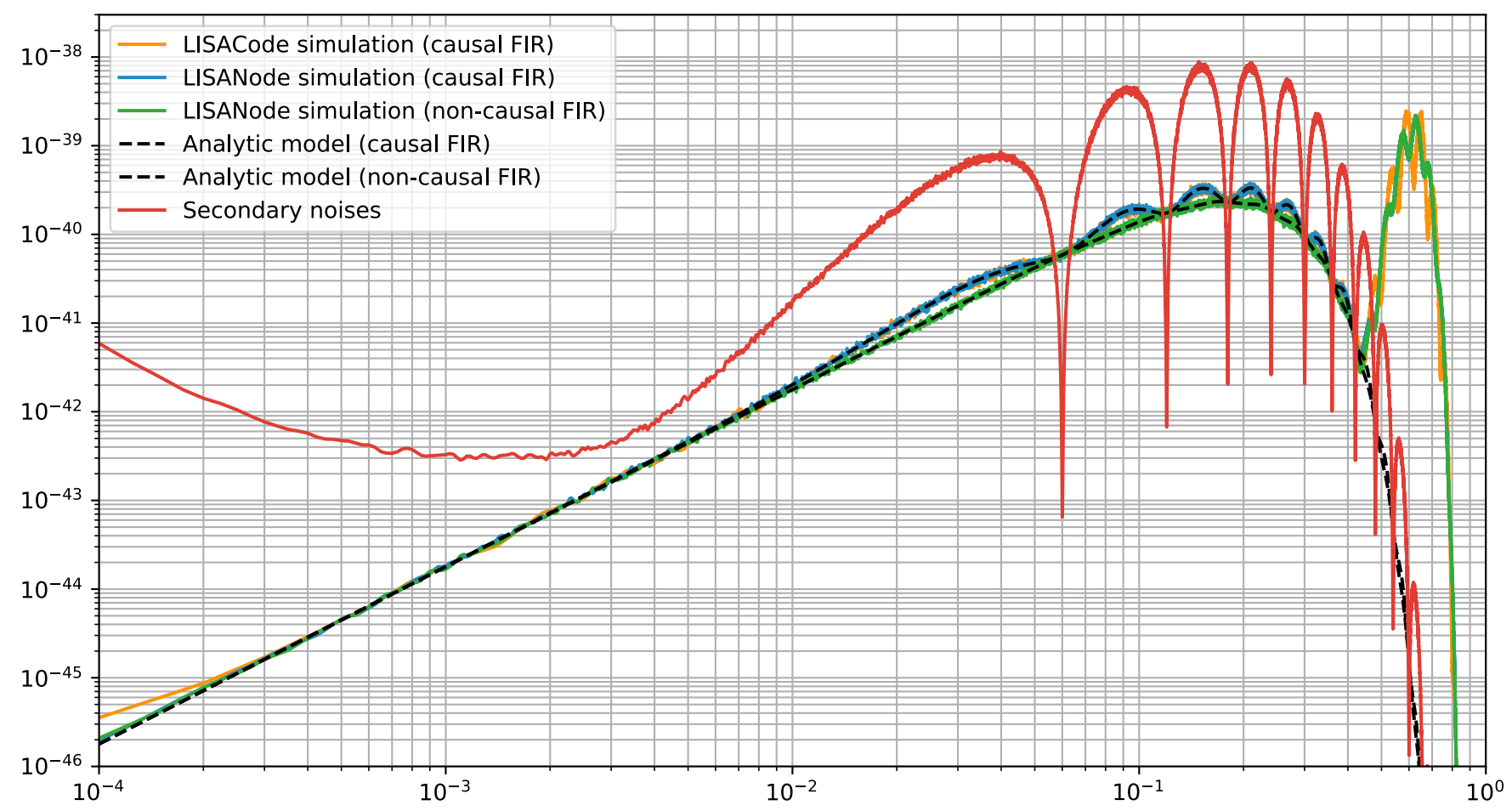

Figure 6. Power spectral density of the residual laser frequency noise in the Michelson $X_{1}$ channel. The LISACode and LISANode simulations use realistic Keplerian orbits, while the theoretical model uses armlengths varying linearly with time. Secondary noises are shown in red and indicate the target level of laser frequency noise suppression.

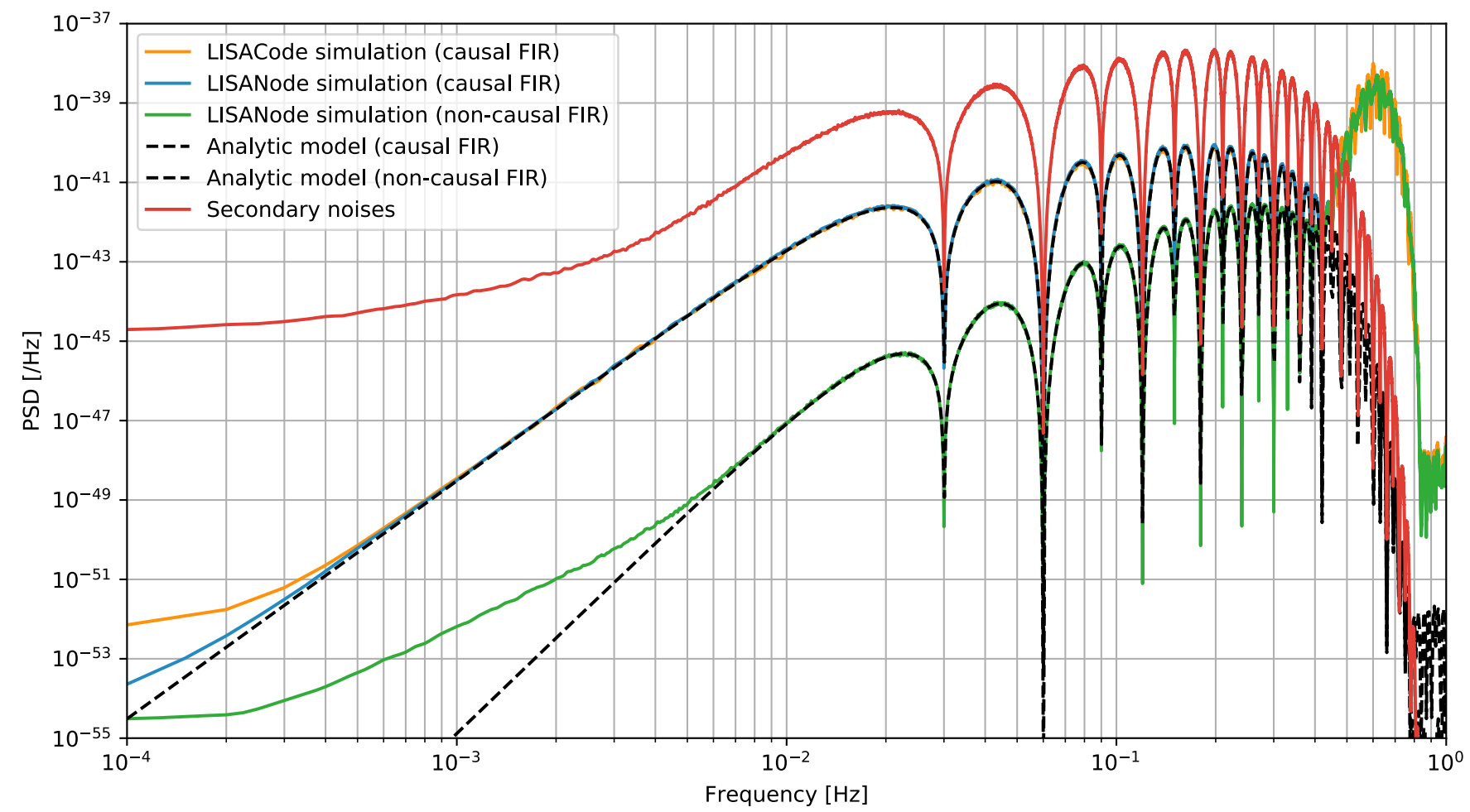

Figure 7. Power spectral density of the residual laser frequency noise in the Michelson $X_{2}$ channel. The LISACode and LISANode simulations use realistic Keplerian orbits, while the theoretical model uses armlengths varying linearly with time. Secondary noises are shown in red and indicate the target level of laser frequency noise suppression. 
Figure 6 shows that first-generation Michelson variables reduce the laser frequency noise down to the required level, but only marginally. This is especially true with causal filters. On the contrary, fig. 7 shows that second-generation Michelson variables can reduce the laser frequency noise up to 3 orders of magnitude below the secondary noises over the entire frequency range, if we use noncausal filters.

In the case of time varying orbits, and in the presence of antialiasing filters, TDI 2.0 is therefore necessary and sufficient to suppress laser frequency noise levels down to mission requirements [1]. Moreover, using noncausal filters allows for comfortable margins.

\section{B. Filter group delay}

TDI uses as inputs the interferometric signals from the six optical benches $\left[s_{i}(t), \tau_{i}(t)\right.$ and $\left.\epsilon_{i}(t)\right]$, and the ranging estimates $L_{i}(t)$ for all six links. A causal filter has a nonvanishing group delay $N / f_{s}$; since it is only applied on the interferometric signals, the latter will be timeshifted while ranging estimates are left untouched. Let us denote the filter group delay operator $\mathcal{D}_{\mathcal{F}}$. As part of the TDI algorithm, one computes terms of the type $\mathcal{D}_{i} \mathcal{D}_{\mathcal{F}} p(t)=p\left(t-N / f_{s}-L_{i}(t)\right)$ when one really wants $\mathcal{D}_{\mathcal{F}} \mathcal{D}_{i} p(t)=p\left(t-N / f_{s}-L_{i}\left(t-N / f_{s}\right)\right)$, or, equivalently, $p\left(t-L_{i}(t)\right)$.

We recognize here an extra noise proportional to the commutator $\left[\mathcal{D}_{\mathcal{F}}, \mathcal{D}_{i}\right] p(t) \approx \dot{L}_{i}\left(N / f_{s}\right) \frac{\mathrm{d} p}{\mathrm{~d} t}\left(t-L_{i}-N / f_{s}\right)$; see eq. 16. It is non vanishing in the case of timevarying armlengths, which explains this flexing-filtering coupling.

\section{Implementation of noncausal filters}

Using eq. 28, we can relate the transfer function $\widetilde{f}_{\text {causal }}$ of the causal version of a filter, to that of the noncausal version $\widetilde{f}_{\text {acausal }}$,

$$
\widetilde{f}_{\text {acausal }}(\omega)=e^{j \omega N / f_{s}} \widetilde{f}_{\text {causal }}(\omega),
$$

and see that the two only differ by a constant delay of an integer number of samples. This delay exactly matches the group delay of the filter. We can therefore deduce output samples of the noncausal filter by simply retimestamping output samples of the causal version such that

$$
y_{k}^{\text {acausal }}=y_{N+k}^{\text {causal }} \text {. }
$$

This relabeling can be performed by the on-board computer, before data are decimated and telemetered to Earth.

One could instead relabel the telemetered interferometric data off-line. This delay is equal to the filter group delay and might not be an integer number of samples anymore due to the downsampling. Therefore, new interpolation errors enter the measurements. Designing the filter such that its group delay is a multiple of the decimated sampling period could solve this issue. This constraint should be accounted for when designing the on-board software.

In the simulations presented in sections $\mathrm{V}$ and $\mathrm{VI}$, we used the latter implementation.

\section{CONCLUSION}

This article addresses the problem of modeling and simulating the residual laser frequency noise, after TDI has been applied, in a realistic instrumental setup. We have focused our analysis on the first and secondgeneration Michelson $X, Y$ and $Z$ variables, and have included the effect of time-varying armlengths, as well as the effects of the on-board antialiasing filters. In our LISACode and LISANode simulations, the armlengths vary according to Keplerian orbits. In the analytic expressions of the residual laser frequency noise spectrum we derive, armlengths vary linearly with time. This is a very good approximation of Keplerian orbits on the time scales of interest. The resulting expressions are functions of both the varying armlengths and of the filter coefficients, and show at leading-order that a new flexingfiltering coupling noise enters the measurements, degrading the expected TDI performance.

We showed that the simulated data match the analytic model with exquisite precision over a large fraction of the LISA frequency band. As a benchmark for the performance of TDI, we used LISANode simulations that include secondary noise only. In the case of time-varying armlengths, TDI 1.5 is shown not be able to achieve sufficient laser frequency noise reduction over the entire frequency range of interest. On the other hand, TDI 2.0 reduces laser frequency noise to well below the secondary noise level, for the case of a standard finite-impulse response filter. TDI 2.0 is therefore the minimal viable TDI generation for LISA.

As demonstrated in this paper, our analytic model and simulations help gain insight into TDI and the various parameters that play a key role in its performance. In particular, we were able to demonstrate that a noncausal filter improves TDI performance and helps reduce further the residual laser noise down to 3 extra orders of magnitude in the middle of LISA frequency band. This noncausal filter can be synthesized using its causal version on board and adapt the TDI algorithm by time-shifting the interferometric signals with respect to the ranging estimates. This concept was demonstrated by our simulations.

One could also use the analytic model developed in this study to optimize the filter coefficients, so that the useful frequency band for data analysis (i.e., the frequency band over which the gravitational signal is not attenuated) is maximized, while the residual laser frequency noise level remains below the secondary noise level. Finally, the effect of other instrumental imperfections and 
artifacts, such as the errors in the absolute ranging or in the interpolation scheme, or even clock noises, remain to be included in both our model and simulations.

\section{Appendix A: Elliptic filter coefficients}

We give the coefficients of the IIR elliptic filter used as a reference in Fig. 3. For numerical stability, the filter is implemented as a series of recursive second-order cells of $z$ transform

$$
\widetilde{f}_{\text {cell }}(z)=\frac{\alpha_{0}+\alpha_{1} z^{-1}+\alpha_{2} z^{-2}}{1+\beta_{1} z^{-1}+\beta_{2} z^{-2}},
$$

and a scaling factor of $2.587527 \times 10^{-4}$ applied at the end of the chain.

First cell: $\alpha=4.882 \mathrm{e}-04,7.239 \mathrm{e}-05,4.882 \mathrm{e}-04, \quad \beta=-1.969$, 9.697e-01. Second cell: $\alpha=9.765 \mathrm{e}-04,-1.487 \mathrm{e}-03,9.765 \mathrm{e}-04, \beta=$ $-1.971,9.719 \mathrm{e}-01$. Third cell: $\alpha=3.125 \mathrm{e}-02,-5.997 \mathrm{e}-02,3.125 \mathrm{e}-02$, $\beta=-1.992,9.961 \mathrm{e}-01$. Fourth cell: $\alpha=7.812 \mathrm{e}-03,-1.402 \mathrm{e}-02$, 7.812e-03, $\beta=-1.974,9.761 \mathrm{e}-01$. Fifth cell: $\alpha=1.562 \mathrm{e}-02$, $-2.980 \mathrm{e}-02,1.562 \mathrm{e}-02, \beta=-1.985,9.886 \mathrm{e}-01$. Sixth cell: $\alpha=$ $1.866 \mathrm{e}+02,-3.501 \mathrm{e}+02,1.866 \mathrm{e}+02, \beta=-1.979,9.818 \mathrm{e}-01$.

\section{Appendix B: FIR filter coefficients}

We give here the $2 N+1$ coefficients $\alpha_{k}$ of the causal and noncausal FIR filters used in Secs. [V] and V] The causal filter's $z$ transform is given by

$$
\tilde{f}_{\text {causal }}(z)=\sum_{k=0}^{2 N} \alpha_{k} z^{-k}
$$

while that of the noncausal filter is

$$
\tilde{f}_{\text {acausal }}(z)=\sum_{k=-N}^{N} \alpha_{N+k} z^{-k}=z^{N} \tilde{f}_{\text {causal }}(z) .
$$

Since the filter is symmetrical, the following holds

$$
\alpha_{k}=\alpha_{2 N-k} \quad \text { for } \quad 0 \leq k \leq 2 N \text {. }
$$

We therefore give half of the coefficients $\alpha_{0} \ldots \alpha_{n}$, the rest can be deduced by symmetry: 0.0225037 , $0.0224708,0.0223744,0.0222146,0.0219926,0.0217101,0.0213692$, $0.0209726,0.0205233,0.0200246,0.0194801,0.0188939,0.0182701$, $0.017613,0.0169273,0.0162175,0.0154884,0.0147444,0.0139904$, $0.0132308,0.0124699,0.0117121,0.0109613,0.0102213,0.00949556$, $0.00878736,0.0080996,0.00743485,0.00679539,0.00618313,0.00559967$, $0.00504627,0.00452385,0.00403305,0.00357419,0.0031473,0.00275216$, $0.0023883,0.00205505,0.00175152,0.00147665,0.00122927,0.00100804$, $0.000811548, \quad 0.000638312,0.00048679,0.000355413,0.000242603$, $0.000146789,6.64234 \mathrm{e}-05,-3.06836 \mathrm{e}-19,-5.39377 \mathrm{e}-05,-9.67836 \mathrm{e}-05$, $-0.000129861,-0.000154416,-0.000171611,-0.000182522,-0.000188139$, $-0.000189361,-0.000187,-0.000181782,-0.000174349,-0.000165266$, $-0.00015502,-0.000144028,-0.000132644,-0.000121158,-0.00010981$, $-9.87885 e-05,-8.82397 e-05,-7.82713 e-05,-6.89586 e-05,-6.03483 e-05$, $-5.24636 e-05,-4.53084 e-05,-3.88703 e-05,-3.31251 e-05,-2.80388 e-05$, $-2.35708 \mathrm{e}-05,-1.96758 \mathrm{e}-05,-1.63062 \mathrm{e}-05,-1.34132 \mathrm{e}-05,-1.09483 \mathrm{e}-05$, $-8.86423 e-06,-7.11608 \mathrm{e}-06,-5.66147 \mathrm{e}-06,-4.46113 \mathrm{e}-06,-3.47914 \mathrm{e}-06$, $-2.68298 \mathrm{e}-06,-2.04359 \mathrm{e}-06,-1.53525 \mathrm{e}-06,-1.13543 \mathrm{e}-06,-8.24623 \mathrm{e}-07$, $-5.86081 \mathrm{e}-07,-4.05589 \mathrm{e}-07,-2.71198 \mathrm{e}-07,-1.72976 \mathrm{e}-07,-1.02753 \mathrm{e}-07$, $-5.38886 \mathrm{e}-08,-2.10473 \mathrm{e}-08,1.67089 \mathrm{e}-22,1.25602 \mathrm{e}-08,1.91793 \mathrm{e}-08$, $2.17835 \mathrm{e}-08, \quad 2.18024 \mathrm{e}-08, \quad 2.02719 \mathrm{e}-08, \quad 1.79219 \mathrm{e}-08, \quad 1.52489 \mathrm{e}-08$, $1.25738 \mathrm{e}-08, \quad 1.00899 \mathrm{e}-08, \quad 7.89963 \mathrm{e}-09, \quad 6.04316 \mathrm{e}-09, \quad 4.52036 \mathrm{e}-09$, $3.30667 \mathrm{e}-09, \quad 2.36459 \mathrm{e}-09, \quad 1.65159 \mathrm{e}-09, \quad 1.12523 \mathrm{e}-09, \quad 7.46354 \mathrm{e}-10$, $4.80713 \mathrm{e}-10, \quad 2.99603 \mathrm{e}-10, \quad 1.79833 \mathrm{e}-10, \quad 1.03279 \mathrm{e}-10, \quad 5.62201 \mathrm{e}-11$, $2.85993 \mathrm{e}-11,1.32841 \mathrm{e}-11,5.3973 \mathrm{e}-12,1.73705 \mathrm{e}-12$.

\section{ACKNOWLEDGMENTS}

This research has been supported by the Centre National d'Études Spatiales, Centrale National pour la Recherche Scientifique (CNRS) and Université ParisDiderot. The development of LISACode and LISANode is part of the LISA Simulation Group activities. We thank Víctor Martín Hernández (IEEC, UAB) for fruitful discussions on the structure of LISANode. We gracefully acknowledge the suggestion by Robert Spero, Kirk McKenzie, Brent Ware, Samuel Francis and Michele Vallisneri (JPL, Caltech) that a noncausal symmetric filter would cancel the residual noise at the leading order. We are also grateful to the members of the LISA Simulation Group for their help in improving the manuscript.
[1] P. Amaro-Seoane et al. (LISA Collaboration), Laser Interferometer Space Antenna, Tech. Rep. (LISA Consortium, 2017) arXiv.org:1702.00786 [astro-ph].

[2] M. Armano and others, Phys. Rev. Lett. 116, 231101 (2016).

[3] M. Armano et al., Phys. Rev. Lett. 120, 061101 (2018).

[4] J. W. Armstrong, F. B. Estabrook, and M. Tinto, ApJ 527, 814 (1999).
[5] M. Tinto and J. W. Armstrong, Phys. Rev. D59, 102003 (1999).

[6] G. de Vine, B. Ware, K. McKenzie, R. E. Spero, W. M. Klipstein, and D. A. Shaddock, Phys. Rev. Lett. 104, 211103 (2010), arXiv:1005.2176 [astro-ph.IM]

[7] T. S. Schwarze, G. Fernández Barranco, D. Penkert, M. Kaufer, O. Gerberding, and G. Heinzel, Phys. Rev. Lett. 122, 081104 (2019), arXiv:1810.00728 [astro- 
ph.IM]

[8] M. Otto, Time-Delay Interferometry Simulations for the Laser Interferometer Space Antenna, Ph.D. thesis, Gottfried Wilhelm Leibniz Universität Hannover (2015).

[9] M. Laporte, H. Halloin, E. Bréelle, C. Buy, P. Grüning, and P. Prat, J. Phys. Conf. Ser. 840, 012014 (2017).

[10] P. Grüning et al., Exp. Astron. 39, 281 (2015).

[11] R. J. Cruz, J. I. Thorpe, M. Hartman, and G. Mueller, Proceedings, 6th International LISA Symposium on Laser interferometer space antenna: Greenbelt, USA, June 1923, 2006, AIP Conf. Proc. 873, 319 (2006).

[12] M. Vallisneri, Phys. Rev. D 71, 022001 (2005), arXiv:grqc/0407102

[13] A. Petiteau, De la simulation de LISA à l'analyse des données, Ph.D. thesis, Université Paris-Diderot (2008).

[14] A. Petiteau, G. Auger, H. Halloin, O. Jeannin, E. Plagnol, S. Pireaux, T. Regimbau, and J.-Y. Vinet, Phys. Rev. D 77, 023002 (2008), arXiv:0802.2023 [gr-qc].

[15] J.-B. Bayle, M. Lilley, and A. Petiteau, "LISANode: A modular simulator for LISA," (2019), to be published.
[16] M. Otto, G. Heinzel, and K. Danzmann, Class. Quant. Grav. 29, 205003 (2012).

[17] M. Tinto and O. Hartwig, Phys. Rev. D 98, 042003 (2018), arXiv:1807.02594 [gr-qc]

[18] S. Barke, Inter-Spacecraft Frequency Distribution for Future Gravitational Wave Observatories, Ph.D. thesis, Gottfried Wilhelm Leibniz Universität Hannover (2015).

[19] S. Dhurandhar, K. Rajesh Nayak, and J. Vinet, Phys. Rev. D 65, 102002 (2002), arXiv:gr-qc/0112059.

[20] K. Rajesh Nayak, S. Koshti, S. V. Dhurandhar, and J.-Y. Vinet, Class. Quant. Grav. 23, 1763 (2006).

[21] S. V. Dhurandhar, K. R. Nayak, S. Koshti, and J.Y. Vinet, Class. Quant. Grav. , 481 (2004), arXiv:grqc/0410093 [gr-qc].

122 LISA Science Study Team, LISA Science Requirements Document, Tech. Rep. 1.0 (ESA, 2018) https://www. cosmos.esa.int/web/lisa/lisa-documents/

[23] S. Babak, J. G. Baker, et al., Class. Quantum Grav. 25, 184026 (2008). 